\title{
Randomised controlled trial of cisapride in preterm infants
}

\author{
R J McClure, J H Kristensen, A Grauaug
}

\begin{abstract}
Aim-To determine the effect of cisapride on gastrointestinal motility in preterm infants.

Methods-Cisapride $(0.2 \mathrm{mg} / \mathrm{kg}, 8$ hourly $)$ or placebo was given first for seven days in a double blind randomised crossover study of 10 preterm infants. Gastrointestinal motility was assessed on day 3 of each treatment. The half gastric emptying time (GET1/2) was determined by using ultrasonography to measure the decrease in the gastric antral cross sectional area after a feed. The whole gastrointestinal transit time (WGTT) was assessed by timing the transit of carmine red through the gut. Treatments were compared using the Wilcoxon matched pairs signed ranks test. Results-Median (range) birthweight was $1200(620,1450) \mathrm{g}$ and postconceptional age $33(29,34)$ weeks at recruitment. GET $1 / 2$ was significantly longer during cisapride treatment than during placebo; the median of the differences $(95 \%$ confidence interval) was 19.2 (11, 30 minutes, $\mathbf{p}=0.008)$. WGTT was also longer during cisapride treatment, but the difference was not significant; the median of the differences was $11(-18,52$ hours, $p=0.1)$. Conclusions-Cisapride delays gastric emptying and may delay WGTT in preterm infants. Its use to promote gastrointestinal motility in this group cannot be recommended.
\end{abstract}

(Arch Dis Child Fetal Neonatal Ed 1999;80:F174-F177)

Keywords: cisapride; gastric emptying; gastrointestinal transit; gastrointestinal motility

Cisapride is a prokinetic agent and stimulates gastrointestinal motility. It is chemically related to metoclopramide, but is largely devoid of central depressant or anti-dopaminergic effects. In adults and children cisapride can increase lower oesophageal sphincter pressure and oesophageal motility, hasten gastric emptying, and accelerate small intestinal and colonic transit of luminal contents in both healthy people and patients with gastrooesophageal reflux..$^{1-7}$ It can also improve coordination of gastric, pyloric, and duodenal motor activity. ${ }^{12}$

Cisapride is being increasingly used in preterm infants for the treatment of gastrooesophageal reflux, apnoea of prematurity resistant to methylxanthines, where silent gastro-oesophageal reflux is a possibility, and for poor feed tolerance due to gastroparesis or impaired intestinal motility. Cisapride is effec- tive in improving gastro-oesophageal reflux in infants. ${ }^{8-10}$ However, we are aware of no randomised controlled study that has objectively examined the effect of cisapride on gastric emptying or intestinal motility in preterm infants. This is despite the known immaturity of gastrointestinal motor activity in this patient group..$^{112}$

This study aimed to determine whether cisapride accelerates gastric emptying and whole gastrointestinal transit time (WGTT) in preterm infants.

\section{Methods}

The study was of a double blind, randomised crossover design. Consecutive very preterm infants (less than 32 weeks of gestation) admitted to the neonatal unit at King Edward Memorial Hospital, Perth, Western Australia, where a clinical decision was made to treat with cisapride, were eligible for the study. Cisapride was prescribed for clinically diagnosed gastrooesophageal reflux or poor feed tolerance, defined for the purpose of this study as a total gastric aspirate volume of at least half of enteral intake over 24 hours. Infants were excluded from the study if they had a congenital anatomical gut malformation or were receiving other drugs known to substantially affect gastrointestinal motility. Treatment with caffeine was not an exclusion criterion as its use was routine to prevent apnoea.

Using previous data on gastric emptying and WGTT, ${ }^{13-16}$ a power calculation (type I error $5 \%$, type II error $20 \%$ ) established that 10 infants should be studied to detect a $25 \%$ difference in both measured outcomes of gastrointestinal motility.

On recruitment, infants were allocated according to a predetermined list of random numbers by the hospital pharmacy department to receive either seven days of cisapride treatment of $0.6 \mathrm{mg} / \mathrm{kg} /$ day in three divided doses given orally followed by seven days of a placebo (hydroxy propyl-methyl cellulose) of equivalent volume, or vice versa. The hospital pharmacy supplied cisapride and placebo preparations of identical appearance and the investigators and staff were blind to infant allocation until after the study was complete.

Gastric emptying and WGTT were measured on the third day of each treatment. Gastric emptying was assessed ultrasonically by serially measuring the change in the gastric antral cross sectional area (ACSA) following a feed, using a previously described method. ${ }^{15}$ ACSA was measured by obtaining real time ultrasound cross sectional images of the gastric antrum with the infant lying in the right lateral 
position. Vertebral bodies, the aorta, and the superior mesenteric artery were used as constant landmarks to ensure the same cross sectional plane of the gastric antrum was measured on each occasion. Images were obtained using a Diosonics Ultrasound Imaging System with a $7.5 \mathrm{MHz}$ probe. Software available on the machine allowed images to be frozen on the screen, the gastric antrum cross sectional circumference to be marked with a steerable cursor, and the ACSA to be calculated.

Before each study the stomach was aspirated using a nasogastric feeding tube. The ACSA was measured before the test feed. A bolus feed of expressed breast milk or preterm formula (S26 low birthweight formula, Wyeth), depending on maternal choice, was given over 10 minutes. Immediately the feed was completed the ACSA was remeasured. The ACSA was subsequently measured at 10 minute intervals until 30 minutes had elapsed, and then continued at 15 minute intervals until 2 hours after the feed, or the prefeed value was reached. At the second measurement each baby received the same volume and type of milk feed as had been received during the first. Treatments were compared by calculating the half gastric emptying time $\left(\mathrm{GET}^{1} / 2\right)$, defined as the time taken for the ACSA to decrease by half of the maximum change seen during gastric filling.

WGTT was measured by timing the transit of the marker carmine red through the gastrointestinal tract. Carmine red (125 mg) was given via a nasogastric feeding tube over 1 minute and the time recorded. At each subsequent nappy change any faeces were inspected for evidence of the dye. WGTT was defined as the elapsed time between carmine red administration and its first appearance in the faeces. To minimise any effect of circadian rhythm, carmine red was always given immediately after a feed as near to 1500 hours as possible. Nappy changes were at the discretion of the attending nurse but were generally 3 to 4 hourly.

Statistical analysis was performed using the Wilcoxon Signed Rank test for paired nonnormally distributed data. Informed parental consent was obtained before each study. The study was approved by the local research ethics committee.

\section{Results}

All 10 infants completed the study without any apparent adverse effects. Four infants were prescribed cisapride for gastro-oesophageal reflux and six for poor milk tolerance. Four were boys. Five infants were randomised to receive cisapride first. Median (range) gestation was $28.5(24,31)$ weeks while birthweight was $1200(620,1450)$ g. Median postnatal age and postconuptional age at first examination was $22(9,38)$ days and $33(29,34)$, weeks respectively. Five infants received fortified expressed breast milk, five preterm formula. Median feed volume was $32(7,75) \mathrm{ml}$.

In every infant $\mathrm{GET}^{1 / 2}$ was longer during cisapride treatment (fig 1). During cisapride treatment and placebo the median (interquar-

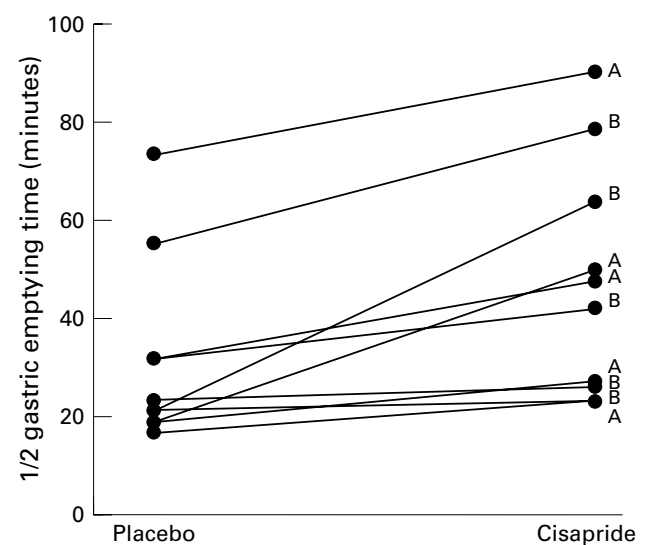

Figure 1 Half gastric emptying times during cisapride and placebo treatments. Paired values for each infant are indicated by horizontal lines. Letter A indicates that infant was randomly allocated to receive cisapride first, letter $B$ indicates placebo.

tile range) $\mathrm{GET}^{1 / 2} / 2$ was $45.6(27,59)$ minutes and $17.4(16,29)$ minutes, respectively, median difference ( $95 \%$ confidence interval) was 19.2 ( 11,30 minutes); $\mathrm{p}=0.008$.

The WGTT was longer in seven infants when measured during cisapride treatment. The median (interquartile range) WGTT was longer during cisapride, 39 (25, 94) hours compared with $32(18,41)$ hours after placebo, but the difference was not significant; median difference (95\% confidence interval) was 11 $(-18,52)$ hours; $\mathrm{p}=0.1$.

\section{Discussion}

Mature gastrointestinal motility depends on a complex interaction of neural and hormonal control mechanisms. This is altered by external factors such as enteral feeding and illness. In preterm infants motility is also affected by gastrointestinal immaturity. The upper gastrointestinal tract displays two different functional states: the interdigestive or fasting state, and the fed or postprandial state. The fasting state is characterised by a propagative cyclical motor pattern, the migrating motor complex (MMC). The MMC is absent before 27 weeks after which it steadily develops, with mature patterns appearing only between 34 weeks and term. ${ }^{11}{ }^{12}$ Gestational age at birth does not seem to influence the rate of development. The fed state is also less well developed in the duodenum in preterm compared with term infants. ${ }^{17}$ Not surprisingly, both gastric emptying and whole intestinal transit have been shown to be delayed in preterm infants. ${ }^{14} 1819$

In view of the immaturity of gastrointestinal motility in preterm infants it was not unexpected that, unlike in infants and adults, no acceleration of gastric emptying and intestinal transit was seen. However, it was surprising to find that cisapride actually retarded gastric emptying. The finding that in seven infants intestinal transit was slower during cisapride treatment, suggests that it may also be delayed.

Only one previous published study by Melis and Janssens ${ }^{20}$ was identified as having examined the effect of cisapride on gastric emptying in preterm infants. In 20 preterm infants (26 to 34 weeks of gestation) gastric emptying was 
improved after 48 hours of cisapride treatment, as assessed by the volume of gastric residuals aspirated during continuous feeding. Comparison with the current study is difficult as theirs was an uncontrolled unblinded observational study and an indirect measurement of emptying was made. Lander et $a l^{1}$ showed that cisapride improved the time to a first sustained milk feed in 11 neonates with prolonged postoperative ileus compared with 12 who received placebo. However, the mean gestation for these infants was 37 weeks and the reason for their impaired gastrointestinal motility was not principally immaturity. In a recent randomised controlled study of 34 infants of less than 32 weeks of gestation, the time taken to tolerate full enteral feeding was unaltered by cisapride compared with placebo, although large gastric residuals did seem to be significantly reduced. ${ }^{22}$ The techniques used in the current study to measure gastric emptying and WGTT have both been validated and used before successfully to assess preterm infants and seem to be reliable. ${ }^{13-16}$

The result might have been affected if the infants of greater prematurity had received cisapride rather than placebo first. This does not seem to have happened; the two most premature infants both received placebo first. Data detailing the pharmacokinetics of cisapride in preterm infants are extremely limited but one study has measured its elimination half life as a mean of 1.8 hours (mean gestational age 29.9 weeks, mean age at measurement 57.5 days) which is much shorter than in adults (published in abstract form only). The "wash out period" after the change over of treatments of more than 48 hours before motility was assessed, therefore, seems to have been adequate.

The mode of action of cisapride is to stimulate serotonergic 5-hydroxytryptamine (5HT4) intestinal receptors, resulting in the release of neurotransmitters in the cholinergic neurones of the gastrointestinal myenteric plexus. ${ }^{23}$ It has been suggested from a study using an animal model by Chen et al that there are 5-HT4 receptors with differing affinities for cisapride. ${ }^{24}$ Significantly, stimulation of high affinity receptors seemed to increase gastrointestinal motor activity while that of low affinity receptors had the opposite effect. We suggest that in the preterm gastrointestinal tract only low affinity receptors have developed sufficiently to be stimulated by cisapride. Alternatively, the dose of cisapride used (currently within quoted recommended ranges) may have been too high for these infants and resulted in stimulation of low affinity receptors. The above study also showed that high doses of cisapride are capable of inhibiting gastrointestinal motor activity.

Several studies have confirmed the efficacy of cisapride in the treatment of gastrooesophageal reflux in infancy. ${ }^{8-10}$ None has examined the same question in infants who are still preterm at assessment. Cisapride has some serious potential side effects in infants, including prolongation of the QT interval which can lead to cardiac arrhythmias. ${ }^{25} 26$
The findings of this study suggest that the use of cisapride cannot be recommended for treatment of gastric stasis or poor intestinal motility in preterm infants. These effects also suggest that cisapride may worsen gastrooesophageal reflux in this population. Until the efficacy of cisapride to treat gastro-oesophageal reflux is determined we believe that this study supports the recent statement made by the United Kingdom Committee on the Safety of Medicines and the Medicines Control Agency: "cisapride is specifically contraindicated in pre term infants." 27

Editor's note: The Medicines Control Agency has stated that cisapride is contraindicated in neonates born before 36 weeks of gestation until they are 3 months of age. This is in addition to the already existing recommendation not to use it in children under the age of 12 years, although the drug is widely used in this age group.

1 Lux G, Katschinski M, Ludwig S, Lederer P, Ellermann A, Domschke W. The effect of cisapride and metoclopramide on human digestive and interdigestive antroduodenal motility. Scand f Gastroenterol 1994;29:1105-10.

2 Johnson AG. The effects of cisapride on antroduodenal co-ordination and gastric emptying. Scand $\mathcal{F}$ Gastroenterol 1989;165:S36-S43.

3 Barone JA, Jessen LM, Colaizzi JL, Bierman RH. Cisapride: a gastrointestinal prokinetic drug. Ann Pharmacother 1994;28:488-500

4 Bergmann JF, Chassany O, Guillausseau PJ, Bayle M, Chagnon S, Caulin C, Sallenave JR. Simultaneous noninvasive evaluation of gastric emptying and orocaecal transit times. Use in studying the actions of cisapride in diabetic patients. Eur f Clin Pharmacol 1992;43:121-4.

5 Bindl L, Buderus S, Ramirez M, Kirchhoff P, Lentze MJ. Cisapride reduces postoperative gastrocaecal transit time after cardiac surgery in children. Intensive Care Med 1996;22:977-80.

6 Cucchiara S, Raia V, Minella R, Frezza T, De Vizia B, De Ritis G. Ultrasound measurement of gastric emptying time in patients with cystic fibrosis and effect of ranitidine on delayed gastric emptying. F Pediatr 1996;128:485-8.

7 Madsen JL Effects of cisapride on gastrointestinal transit in healthy humans. Dig Dis Sci 1990; 35:1500-4.

8 Vandenplas Y, Deneyer M, Verlinden M, Aerts T, Sacre L. Gastroesophageal reflux incidence and respiratory dysfunction during sleep in infants: treatment with cisapride. 7 Pediatr Gastroenterol Nutr 1989;8:31-6.

9 Carroccio A, Iacono G, Li Voti G, et al. Gastric emptying in infants with gastroesophageal reflux. Ultrasound evaluation before and after cisapride administration. Scand $\mathcal{f}$ Gastroenterol 1992;27:799-804.

10 Greally P, Hampton FJ, MacFadyen UM, Simpson H. Gaviscon and Carobel compared with cisapride in gastroGaviscon and Carobel compared with cisapride in gast

11 Bisset WM, Watt J, Rivers RPA, Milla PJ. Ontogeny of fasting small intestinal motor activity in the human infant. Gut 1988;29:483-8.

12 Berseth CL. Gestational evolution of small intestine motility in preterm and term infants. F Pediatr 1989;115:646-51.

3 Rubaltelli FF, Largajolli G. Effect of light exposure on gut transit time in jaundiced newborns. Acta Paediatr Scand 1973;62:146-8.

14 Ernst JA, Rickard KA, Neal PR, Pao-Lo Y, Oei TO, Lemons JA. Lack of improved growth outcome related to non-nutritive sucking in very low birth weight premature infants fed a controlled nutrient intake: A randomised prospective study. Pediatrics 1989;83:706-16.

15 Newell SJ, Chapman S, Booth IW. Ultrasound assessment of gastric emptying in the preterm infant. Arch Dis Child

16 McClure RJ, Newell SJ. The effect of fortifying breast milk on gastric emptying. Arch Dis Child 1996;74: F60-2.

17 Al Tawil YS, Berseth CL. Gestational and postnatal maturation of duodenal motor responses to intragastric feeding. F Pediatr 1996;129:374-81

18 Gupta M, Brans YW. Gastric retention in neonates. Pediatrics 1978;62:26-9.

19 Cavell B. Reservoir and emptying function of the stomach of the premature infant. Acta Paediatr Scand 1982;296: 60-1.

20 Melis K, Janssens G. Long-term use of cisapride (Prepulsid) in premature neonates. Acta Gastroenterol Belg in premature

21 Lander A, Redkar R, Nicholls G, et al. Cisapride reduces neonatal postoperative ileus: randomised placebo controlled trial. Arch Dis Child 1997;77: F119-22.

22 Enriquez A, Bolisetty S, Patole S, Garvey PA, Campbell PJ. Randomised controlled trial of cisapride in feed intolerance in preterm infants. Arch Dis Child 1998;79:F110F13. 
23 Pan H, Galligan JJ. 5-HT1A and 5-HT4 receptors mediate inhibition and facilitation of fast synaptic transmission in 24 Chen HT, Goh MH, Pan S. The effect and mechanism of the prokinetic action of cisapride on gastrointestinal 25 Lewin MB, Bryant RM, Fenrich AL, Grifka RG. Cisaprideinduced long QT interval. $₹$ Pediatr 1996;128:279-81.
in 1 .
26 Dieckmann K, Maurage C, Rolland JC, Ramponi N, Jonville AP. Torticollis as a side effect of cisapride treatment in an infant. $\mathcal{F}$ Pediatr Gastroenterol Nutr 1996;22:336.

27 Committee on the Safety of Medicines/Medicines Control Agency. Cisapride (Prepulsid): Risk of arrhythmias. Current Problems in Pharmacovigilance 1998;24:11. 